\title{
Band 25, Heft 4, August 2013
}

Editorial

201 Traditionelle Europäische Medizin (TEM) und Traditionelle Europäische Naturheilkunde (TEN) - brauchen wir mehrere Begriffe? Uehleke, B. (Zürich/Berlin)

Expertengespräch

204 Wer Phytotherapeutika entwickelt, hat den ganzen Planeten zur Verfügung

Klaffke, O. (Olten)

Wissenstransfer

208 Sind komplementärmedizinische Behandlungen preiswerter?

Uehleke, B. (Zürich/Berlin)

Kommentar

210 Qui bono? - Zu Kendall DE: «National Center for Complementary and Alternative Medicine (NCCAM) Introduction to Acupuncture with Disclaimer». Schweiz Z Ganzheitsmed 2013;25:

DOI: 10.1159/000353908

Ausfeld-Hafter, B. (Bern)

\section{Ganzheitsmedizin aktuell}

Fortbildung

212 Phyto- und Mykotoxine (4): Psilocybin

Jeannin, J.-M. (Basel)

214 Anthroposophische Medizin

Ganz, C. (Zürich)

217 Taiji - Ressourcenaktivierung und Stressprotektion durch Ruhe in Bewegung

Nedeljković, M. (Zürich/Bern)
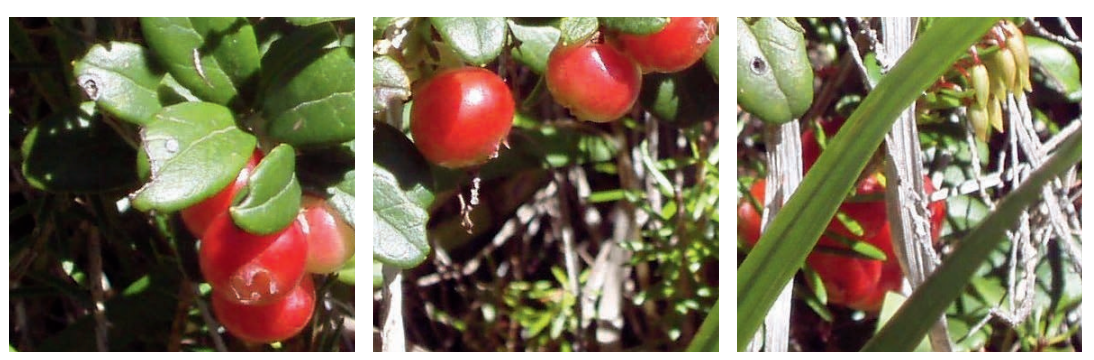

Der hier abgebildete Preiselbeerstrauch (Vaccinium vitisdaea (Ericaceae)) ist ein illustratives Beispiel für die Anpassung einer Pflanze an höhere Lagen: Niedriger Wuchs und eine Beschränkung auf ein halbes Dutzend Laubblätter ermöglichen eine perfekte Anpassung an die Anforderung des hochalpinen Standorts. Die Preiselbeeren werden in der Küche verwendet; die Früchte und die Blätter haben als Heilmittel zur Behandlung von urologischen Problemen Eingang gefunden (V. vitis-idaea, gefunden auf dem Weg nach Tschlin GR auf ca. $1600 \mathrm{~m}$ 
220 Ärztekongress VegMed 2012: Vegetarische Ernährung und Medizin solide Evidenz, aber bessere Umsetzung in die Praxis nötig

Gehrke, M. (Essen)

223 Chronisch-entzündliche Darmerkrankungen aus der Sicht der Komplementärmedizin - Teil 2

Winnicki, C.; Orzessek, B. (Brunnen)

\section{Circadiane Rhythmen}

Ganz, C. (Zürich)

\section{Essays}

231 Zur Relevanz wissenschafts-, medizin- und sozialgeschichtlicher Grundlagenforschung

Relevance of Basic Research in the History of Science, Medicine, and Sociology

Sur la pertinence de la recherche fondamentale en histoire de la science, de la médecine et de l'histoire sociale

Schmidt, J.M. (München)

241 National Center for Complementary and Alternative Medicine (NCCAM) Introduction to Acupuncture with Disclaimer

Die «National Center for Complementary and Alternative Medicine (NCCAM) Introduction to Acupuncture» mit Disclaimer

Centre national de Médecine complémentaire et alternative (NCCAM) Introduction à l'acupuncture avec note de non-responsabilité

Kendall, D.E. (Agoura Hills, CA)

247 Bildungsmarkt

248 Buchbesprechungen

250 Tagungen und Kongresse

Mitteilungen UNION

254 Zum Gedenken an Andreas Beck

Ausfeld-Hafter, B. (Bern)

Mitteilungen UNION

256 Kenntnisse in Komplementärmedizin für Studierende der Medizin und Pharmazie

229 Impressum

U3 Hinweise für Autoren
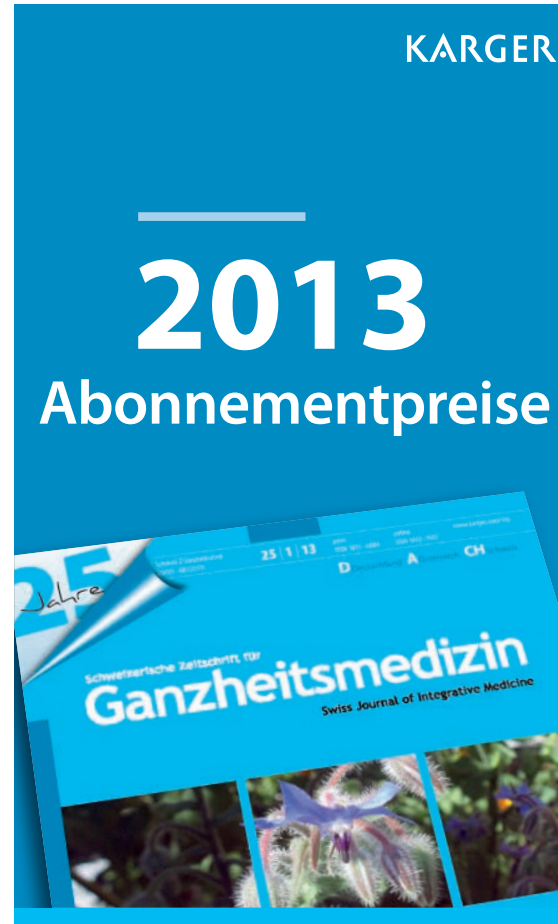

Band 25 mit 6 Heften

-Print EUR 63,- / CHF 75,-

- Online EUR 63,- / CHF 75,-

-Kombi EUR 78,- / CHF 94,-

Versandkosten für Print und

Kombi-Abonnement

EUR 25,- / CHF 30,-

Preise für Gesellschafts-

mitglieder auf Anfrage

Unseren Abo-Service erreichen

Sie unter:

t +49 76145 207-21, f-14

e.traenkle@karger.com

Besuchen Sie unsere Homepage:

www.karger.com/szg 